\title{
CANCER OF THE RECTUM
}

\author{
By Ronald W. Raven, O.B.E., F.R.C.S.
}

Surgeon to the Royal Cancer Hospital (Free), Assistant Surgeon to the Gordon Hospital for Diseases of Rectum and Colon, Surgeon to Out-Patients, the French Hospital

\section{General Features of the Disease}

Cancer of the rectum is seen frequently in this country and it is one of the commonest forms of malignant disease, accounting for a total number of more than 6,000 deaths during the year 1942. The number of deaths occurring during 1942 as a result of cancer in the common sites is shown in Table $\mathbf{r}$.

TABLE I

CANCER AND OTHER MALIGNANT TUMOURS. DEATHS FROM CERTAIN SITES.

(Registrar-General's Statistical Review of ENGLAND AND WALES, 1942)

\begin{tabular}{|c|c|c|}
\hline \multirow{2}{*}{ Site of Disease } & \multicolumn{2}{|c|}{ NuMber OF DEATHS } \\
\hline & Male & Female \\
\hline $\begin{array}{cccc}\text { Digestive organs } & \text { and } & \text { peri- } \\
\text { toneum } & \ldots & \ldots & \ldots\end{array}$ & 19,413 & 17,055 \\
\hline Oesophagus $\ldots \quad \ldots$ & $\mathbf{I}, \mathbf{5 8 4}$ & $68 \mathrm{r}$ \\
\hline Stomach and duodenum & 7,151 & 5,586 \\
\hline $\begin{array}{c}\text { Intestines other than duo- } \\
\text { denum and rectum }\end{array}$ & 4,432 & 5,453 \\
\hline Rectum & 3,698 & 2,442 \\
\hline Liver and Biliary passages .. & 1,249 & 1,625 \\
\hline Pancreas & 1,146 & I, 056 \\
\hline Breast .. & 60 & 7,203 \\
\hline$\cdot \ldots$ & - & 4,575 \\
\hline Specified as cancer of cervix & - & $\mathrm{I}, 884$ \\
\hline $\begin{array}{cccc}\text { Other or unspecified cancer of } \\
\text { uterus } & \cdots & \cdots & \ldots \\
\end{array}$ & - & 2,691 \\
\hline Respiratory System .. & 5,596 & $\mathbf{1 , 5 2 5}$ \\
\hline Prostate & 2,388 & $\longrightarrow$ \\
\hline Urinary organs & $\mathbf{1}, 6 \mathbf{2 3}$ & 907 \\
\hline
\end{tabular}

The figures in Table I show that males are affected more frequently than females, there being 3,698 deaths in males and 2,442 in females.

The age incidence is shown in Table 2.

\section{TABLE II}

CANCER OF RECTUM. AGE INCIDENCE.

(Registrar-General's Statistical Review of O ENGLAND AND WALES, 1942)

\begin{tabular}{|c|c|c|c|}
\hline \multirow{2}{*}{ Ages } & \multirow{2}{*}{ AT Death } & \multicolumn{2}{|c|}{ Sex } \\
\hline & & Males & Females \\
\hline \multirow{12}{*}{. } & $20-$ & 5 & 7 \\
\hline & $25-$ & 14 & 8 \\
\hline & $30-$ & 19 & 29 \\
\hline & $\begin{array}{l}35- \\
40-\end{array}$ & $\begin{array}{l}47 \\
68\end{array}$ & $\begin{array}{l}45 \\
80\end{array}$ \\
\hline & $\begin{array}{l}45- \\
50-\end{array}$ & $\begin{array}{r}92 \\
185\end{array}$ & $\begin{array}{l}114 \\
182\end{array}$ \\
\hline & $55-$ & 346 & 256 \\
\hline & $60-$ & 561 & 317 \\
\hline & $65-$ & 806 & 414 \\
\hline & $70-$ & 788 & 412 \\
\hline & 75 - & 500 & 336 \\
\hline & $80-$ & 200 & 159 \\
\hline & $85-$ & 67 & 83 \\
\hline
\end{tabular}

The disease is more frequently seen in females under the age of 40 years than in males. Cuthbert Dukes ${ }^{1}$ has called attention to the correlation which exists between the extent of lymphatic metastases and the age of $ᄋ$ the patient, and by analysis it was shown that $\rightarrow$ in patients under the age of 40 years lymphatic metastases were found in $71.8 \pm 4.3$ per cent., $N$ and in the age-group 40 to 59 years they were present in $50.9 \pm 2.4$ per cent. This observation is in line with the general rule that cancer is often more virulent in the young than the old.

\section{Pathological Considerations}

The initial lesion. Carcinoma of the rectum commences in one of two ways, namely, as a 
nodular thickening in the mucous membrane, or as a malignant degenerative process in a pre-existing lesion such as an adenoma or a villous papilloma. In the first type, as the lesion increases in size it becomes a raised swelling which becomes ulcerated at a later stage. It is now a recognized fact that an adenoma or papilloma of the rectum must be regarded as a precancerous lesion and dealt with accordingly.

The author ${ }^{2}$ has reported the case record of a patient with a very early carcinoma of the rectum for which a perineal excision of the rectum was performed following exploratory laparotomy and left inguinal colostomy. The specimen shows a small carcinomatous ulcer $\frac{1}{4}$ in. in diameter with a proliferating edge. Microscopical examination of vertical sections passing through the centre of the small tumour show portions of a columnar celled adenocarcinoma invading the submucous layer. A small group of the tumour cells suriounded by small round cells, evidently representing the base of a crypt in which the epithelium has undergone malignant change, is seen also within the upper limit of the circular muscle layer. Two lymph nodes were present and showed no sign of carcinomatous invasion.

Multiple lesions. The occurrence of multiple carcinomata of the rectum, or of the rectum and colon, is of more than academic interest owing to its frequency. Thus Dukes ${ }^{1}$ in a series of 1,000 cases of carcinoma of the rectum reported the presence of more than one focus of carcinoma in 29 cases (2.9 per cent.); four of these were associated with familial polyposis intestini and in the remainder only two carcinomata were found in each specimen, these were separated by at least an inch or more of normal bowel. It was found that the two growths were usually similar in size and shape and of equal duration, but in others one of the neoplasms was at an earlier stage of development. Gabriel ${ }^{3}$ considers that the incidence of simultaneous double rectal carcinoma is probably more than 5 per cent., and in a series of 100 one stage excisions of the rectum by the perineoabdominal route no less than eight double carcinomata of the rectum itself or of the rectum and pelvic colon were found. The subject has been reviewed by Norbury ${ }^{4}$ who called attention to certain important matters as follows. Multiple polypi are commonly associated with carcinoma of the large bowel and one or more may undergo malignant transformation producing multiple carcinomata. A study of the literature showed that in the majority of cases of multiple malignant growths of the large bowel, the lesions are situated usually in the rectum or pelvirectal region. Norbury stresses the practical implications of the incidence of multiple neoplasms. Thus sigmoidoscopy should be carried out to exclude adenomatosis or the presence of a second neoplasm in the rectum or lower part of the pelvic colon. When an operation is planned for excision of the rectum, or resection of the colon for malignant disease, the possibility that multiple neoplasms may be present must be considered, and he advocates the routine examination of the whole of the colon in such cases through an adequate abdominal incision.

Relation with familial polyposis intestini. This condition is characterized by the presence in the rectum and colon of multiple adenomata, either sessile or pedunculated, and of various sizes. Often the larger neoplasms are in the distal colon and they may project into the rectum. It is recognized that this disease has a familial incidence and it may occur in several generations. A very important aspect is the relationship which exists between this condition and carcinoma. Malignant changes may occur in one or more of these neoplasms and a number of cases have been reported in the literature of multiple carcinomata of the bowel in association with polyposis and the age of such patients may be younger than the normal age at which carcinoma of the rectum ocours.

\section{Types of growth}

There are several types of carcinoma of the rectum which can be distinguished on macroscopic examination. These are described as follows :-

Ulcerative type. In this variety of neoplasm the main feature is ulceration, round or oval in shape. The depth of the ulcer varies, it may penetrate through the entire thickness of the wall of the rectum and involve the perirectal tissues and adjacent structures. The margins of the ulcer are raised, nodular and of 
irregular outline; the central zone of the lesion being excavated and depressed.

Polypoidal type. This variety presents as a large tumour mass which projects into the lumen of the rectum, the surface being somewhat smooth or markedly irregular. The tumour may be pedunculated, semipedunculated or sessile, and various degrees of distension of the lumen of the bowel are seen above the neoplasm. It may spring from one quadrant of the rectum or the whole circumference may be involved in the malignant process. The surface of the tumour may be ulcerated of varying degrees.

Stenotic type. In this variety of neoplasm a hard ring like carcinoma is formed which encircles the wall of the rectum transversely and causes a marked degree of stenosis with dilatation of the bowel above the stricture. There is marked fibrosis in the wall of the rectum involved in the neoplastic process and the growth may show a very moderate degree of surface ulces ation.

Complex type. In certain cases there is a complex tumour mass composed of several types. One portion of the neoplasm is of the polypoid type forming multiple pedunculated papillomata which project into the lumen of the bowel. In another part the features of the ulcerative type may be seen, with raised, nodular margins which are sometimes undermined. In addition the lumen of the bowel may be occluded to varying degrees, the bowel above being distended.

Histologically there are two main types of carcinoma of the rectum, namely the columnar celled type and the mucoid typr. Further, the types of cells vary considerably, in some tumours there is a preponderance of ar,aplastic cells and in others the majority of cells are well aifferentiated. There are also many tumours which contain both varieties of cells in varying proportions. An attempt has been made to apply the histological grading of Broders but this has not been entirely satisfactory as an index of prognosis, it is better to rely on a detailed study of the specimen with special regard to invasion of the extrarectal tissues, regional lymph nodes and the rectal veins as described by Dukes.
The Spread of the Disease

Important contributions to our knowledge $\stackrel{0}{\llcorner}$ concerning the mode of spread of carcinoma of the rectum have been made by Miles ${ }^{5}$ and Dukes. There are three main ways by which the disease progresses which will now be $\frac{\bar{N}}{\bar{N}}$ described.

Spread by direct extension. The neoplasm spreads locally in the mucous and submucous $\stackrel{\infty}{\circ}$ tissues both longitudinally and transversely. Infiltration through the wall of the rectum is $\vec{\omega}$ progressive, each of the components being $\frac{\circ}{\partial}$ penetrated in their turn until the perirectal fatty tissues are involved, and subsequently the fascia propia and neighbouring structures, urinary bladder, prostate, uterus, vagina or 엉 sacrum.

Dukes has introduced a method whereby the $\bigcirc$ degree of local spread can be assessed. $\mathrm{He}-$ distinguishes three groups of cases based on the extent of the disease. In group $A$ are included $\vec{c}$ "those in which the growth is confined to the wall of the rectum and there is no extension to $\overrightarrow{0}$ the extrarectal tissues or regional lymph nodes $\stackrel{\circ}{*}$ In group $\mathbf{B}$.are cases where the growth he spread by direct extension into the extra rectal tissues but there are no metastases in the regional lymph nodes. In group $\mathrm{C}$ are cases where there are metastases in the regional $\frac{}{\circ}$ lymph nodes and in this category are two sub- $\overrightarrow{\vec{B}}$ divisions, namely group C.I in which there is $\frac{}{3}$ at least one lymph node below the point of ligature of the vascular pedicle which is free from carcinoma ; group C.2 in which all lymph nodes as high as the point of ligature of the vascular pedicle are involved by metastases. Dukes found that of the cases of carcinoma of the rectum which were operable, 15 per cent. were in group A, 35 per cent. in group B, and $O$ 50 per cent. in group C.

Spread by the lymphatic system. This is a very important method of dissemination and o except in neoplasms of a high grade of $N$ malignancy, the regional lymph nodes are usually not involved until the carcinoma has ${ }^{\omega}$ invaded the extrarectal tissues. The spread appears to take place slowly from node to node $\frac{\widetilde{\Phi}}{}$ commencing in the retrorectal group upwards $\stackrel{?}{?}$ to the lymphatics of the pelvic mesocolon, then 0 the paracolic nodes, the nodes situated at the $\frac{\vec{D}}{\mathrm{D}}$ bifurcation of the left common iliac artery and $\frac{?}{\mathbb{Q}}$ 
finally the aortic group is affected. Miles has stressed the importance of a thorough removal of the whole of the pelvic mesocolon on the ground that there is scarcely a part of it in which metastases have not been found.

Spread by venous system. Extension of the malignant process through the venous system occurs by a process of thrombosis or embolism. In a series of 539 cases reported by Dukes in which the veins were dissected out and examined microscopically there was evidence of such extension in 17.8 per cent. It seems that invasion of the veins does not occur until the growth has invaded the extrarectal tissues and it is related with spread along the lymphatics. The importance of this method of spread is exemplified by the frequency of metastases in the liver.

\section{The Early Symptomatology}

Success in the treatment of cancer largely depends upon early diagnosis and the institution of treatment during the initial phases in the development of the neoplasm. This statement applies to cancer of the rectum and if the survival rate from this disease is to be improved materially then patients must present themselves for treatment at an earlier stage in the disease. Careful study should be given to the early symptomatology so that in the presence of these indications of serious disease a complete investigation can be carried out. It is a distressing fact that so many patients are in an advanced stage of the disease when they present themselves, rendering the performance of radical surgical extirpation impossible.

The disease is often insidious in onset and there may be a latent period during which the symptoms are minimal or absent altogether. The significance of the first symptom must be recognized in order that a complete investigation may be instituted and the diagnosis made. If some other abnormality of the ano-rectal region is present this must not be allowed to mask the underlying major lesion. For example, a patient may be treated for haemorrhoids and a carcinoma higher in the rectum missed until late in its development. Too much stress has been given to symptoms, such as haemorrhage, alternating diarrhoea and constipation and a change in the size of the stools, in the past, and it is necessary to emphasize that these are often late symptoms or indications that the disease is well established. It is likely that a more detailed enquiry into changes in the function of the bowel will lead to earlier diagnosis in many more patients in the future.

The symptomatology varies with the site of the disease in the rectum but attention is called to certain general early symptoms which may be present wherever the disease is situated.

$A$ change in bowel habit. The significance of a change in bowel habit must be understood clearly, and careful enquiries made concerning the way in which the patient's bowel usually functioned before the change occurred. Thus a patient may state that he had an action of the bowel once or more every day and then observed that he became constipated or that the bowels were loose. In other cases a patient who has been constipated notices that this changes to diarrhoea; on the other hand a patient with loose bowels becomes constipated. Other points to remember when consideration is given to the bowel habits of these patients are :-a change may occur in the timing of defaecation during the day, for example the bowel action occurs later than has been usual ; there may be urgency of defaecation or a sudden desire to defaecate occurs immediately after the ingestion of food.

Haemorrhage from the anus. 'T his may be the first and an early symptom of the disease. In the earliest carcinoma of the rectum which the author has operated on the patient gave the history of slight bleeding from the anus extending over a period of several weeks, but one week before she attended hospital there was an attack of severe bleeding. The bowels were opened regularly with aperients, there was no discharge, diarrhoea or pain. Sigmoidoscopy revealed a small ulcer with a proliferating edge and bleeding occurred when it was touched.

Mucoid discharge. In the early phases of the development of a carcinoma of the rectum there may be a slight discharge of mucous from the anus. This is also present in certain benign conditions such as an adenoma of the rectum.

Pain. This is not a symptom of early carcinoma of the rectum except when the neo- 
plasm involves the anal canal or anus. In these patients there may be an ache in the rectum with sharp pain during defaecation. In the later phases of the disease the pain may be very severe especially when the carcinoma infiltrates through the anterior wall of the rectum into the prostate, urinary bladder or urethra, or when it involves the sacrum.

Correlation of symptomatology and site of carcinoma. The symptomatology of carcinoma of the rectum varies according to its site, so that there is no complete train of symptoms which is applicable to all cases. Thus a carcinoma at the recto-sigmoidal junction tends to be of the stenotic variety and produces symptoms of intestinal occlusion. When the neoplasm is of the proliferating type there is usually a mucoid discharge and diarrhoea. Bleeding may' also occur; later an attack of intestinal occlusion may develop due to faecal impaction above the neoplasm or from intussusception. A carcinoma situated in the ampulla may be present for several months before causing symptoms. When the surface becomes ulcerated, bleeding occurs. When the neoplasm is situated low in the rectum close to the anal canal there may be a sense of fullness persisting in the rectum following defaecation. There is also pain which is experienced during defaecation and this often persists for some time afterwards.

The late symptomatology. When the disease is well-established or advanced the symptoms become more severe. The bowels are very irregular with severe diarrhoea and the passage of blood and mucous. There may be incontinence or faeces and a marked discharge from the anus. The patient may complain of the sensation of a tumour in the rectum. Pain becomes severe and may be experienced in several situations including the ano-rectal region, the lower part of the back, the perineum or abdomen. Pain may be referred to the lower extremity. The general condition of the patient deteriorates fairly rapidly, wasting and secondary anaemia soon develop. Inflammatory complications may occur including the development of an ischiorectal abscess and an ano-rectal fistula.

Physical examination. An assessment is made of the general physical condition of the patient including the nutrition, cardio- vascular, respiratory and urinary systems. In certain cases there is extension of the disease to the left supraclavicular lymph nodes. The abdomen is examined critically to determine whether there are any abdominal extensions of the disease especially to the liver or regional lymph nodes, or secondary deposits in the great omentum and peritoneum. Any abdominal distension is noted and the condition of the inguinal lymph nodes is determined. Routine matters such as oral sepsis are dealt with as in any surgical patient.

Digital examination of the rectum is carried out with the patient in the right lateral position and every segment of the rectum palpated critically with the index finger. If a neoplasm is palpable, its type, extent, size and induration, together with the degree of mobility or fixity which is present are determined. Enlarged lymph nodes in the mesorectum will be felt posteriorly or posterolaterally.

Proctoscopy and sigmoidoscopy. These examinations must always be carried out so that the carcinoma can be visualized and the finde ings on digital examination can be confirmede When the sigmoidoscope can be introduce into the rectum at a higher level thar the carcinoma, the bowel can be inspected for the presence or other abnormalities such as an adenoma, polyp or a second carcinoma. Many cases or carcinoma high in the rectum which are beyond the reach of the index finger will be discovered by the use of the sigmoidoscope.

Biopsy. The removal of a small portion of a carcinoma of the rectum can be done with Brüning's forceps through the sigmoidoscope. By this means the diagnosis can be confirmed.

Radiological examination after a barium enema. This method of investigation is unnecessary in cases of carcinoma of the rectum but it is sometimes useful in rectosigmoidal carcinoma when the growth is not seen with the sigmoidoscope which cannot reach this region on account of spasm or kinking of the bowel. This region of the bowel is difficult to visualize radiologically and the examination must be carried out by an expert radiologist. It is pointed out that a negative examination does not exclude the presence of a carcinoma. When a malignant neoplasm is present it will give rise to a filling defect in the bowel or a stricture will be seen. 
Treatment. The only method of treatment available at present for the cure of carcinoma of the rectum is radical surgical excision. Discussing this matter Miles ${ }^{5}$ states that the rectum is especially amenable to radical extirpation for malignant disease, owing to the fact that the main line of advance in the invasion of the extra-mural tissues takes place in structures which are not only removable, but are also remote from the primary disease necessitating the lapse of a certain length of time before the invading cells have progressed beyond the limits of the field of possible operation. Indeed, Miles considers that no other organ in the body is so favourably placed in this respect. The radical operation for carcinoma of the rectum must be planned with regard to the spread of the disease to structures removed from the primary site. It must embrace all the tissues which pathological studies have shown to be prone to carcinomatous invasion. In describing the evolution of the abdomino-perineal excision of the rectum, Miles, who so brilliantly conceived and executed it, stated he was impressed by the frequency of recurrences after perineal excision operations and was convinced that the fault lay in technique and probably in conservatism in the removal of the tissues surrounding the diseased portion of the bowel.

At the present time there are two standard operations for the radical excision of the rectum for carcinoma, namely, the abdominoperineal operation (Miles) and the perineoabdominal operation in one stage developed by Gabriel. Both operations may be carried out as two-stage procedures if it is necessary owing to the poor general condition of the patient. There are certain disadvantages associated with a two-stage operation, chiefly due to the presence of a colostomy and the risk of septic complications developing in consequence.

The synchronous abdomino-perineal excision operation. During recent years this modification has been developed and is practised frequently. In this method two surgeons are operating at the same time, one carrying out the abdominal, and the other performing the perineal part of the procedure. By this technique the time taken for the operation is diminished and the average case should be completed in one hour. There is another important and distinct advantage, however, namely in the case of a carcinoma of doubtful operability due to local fixation it is most helpful for one surgeon to separate the growth from surrounding tissues working from the abdominal aspect and the other surgeon to do the same from the perineal aspect. Using this technique it is probable that neoplasms of border-line operability can be removed satisfactorily.

\section{Conservative Resections of the Rectum with Preservation of the Sphincters}

From time to time there has been an advocacy of these operations for certain cases of early carcinoma of the rectum. The object of these procedures is to avoid the establishment of a permanent colostomy. A number of successful conservative resections have been reported in the literature, the patients remaining alive and free from recurrent disease over a large number of years. There are two chief methods, namely the Kraske operation embodying sacral excision with conservation of the sphincters and circular rectorrhaphy, and the combined abdomino-sacral excision. On general principles.it appears sounder to perform a radical excision of the rectum with every prospect of cure than to temporize with a conservative operation and run the risk of recurrent carcinoma in an early case.

\section{The Abdomino-Perineal Excision of the Rectum}

The technical details of this operation, so ably worked out and described by Miles, are well-known and widely applied. The operation is of considerable magnitude and no effort must be spared in the pre- and post-operative treatment if a successful result is to be achieved. During recent years therc has been a fall in the operative mortality and due credit is given to the contribution made by this treatment before and after operation which will be described.

Pre-operative care. The patient is admitted to hospital for a period of ten days betore the operation is performed to allow investigatior's and treatment to be carried out including an assessment of the patient's general health and nutritional state. If protein deficiency and 


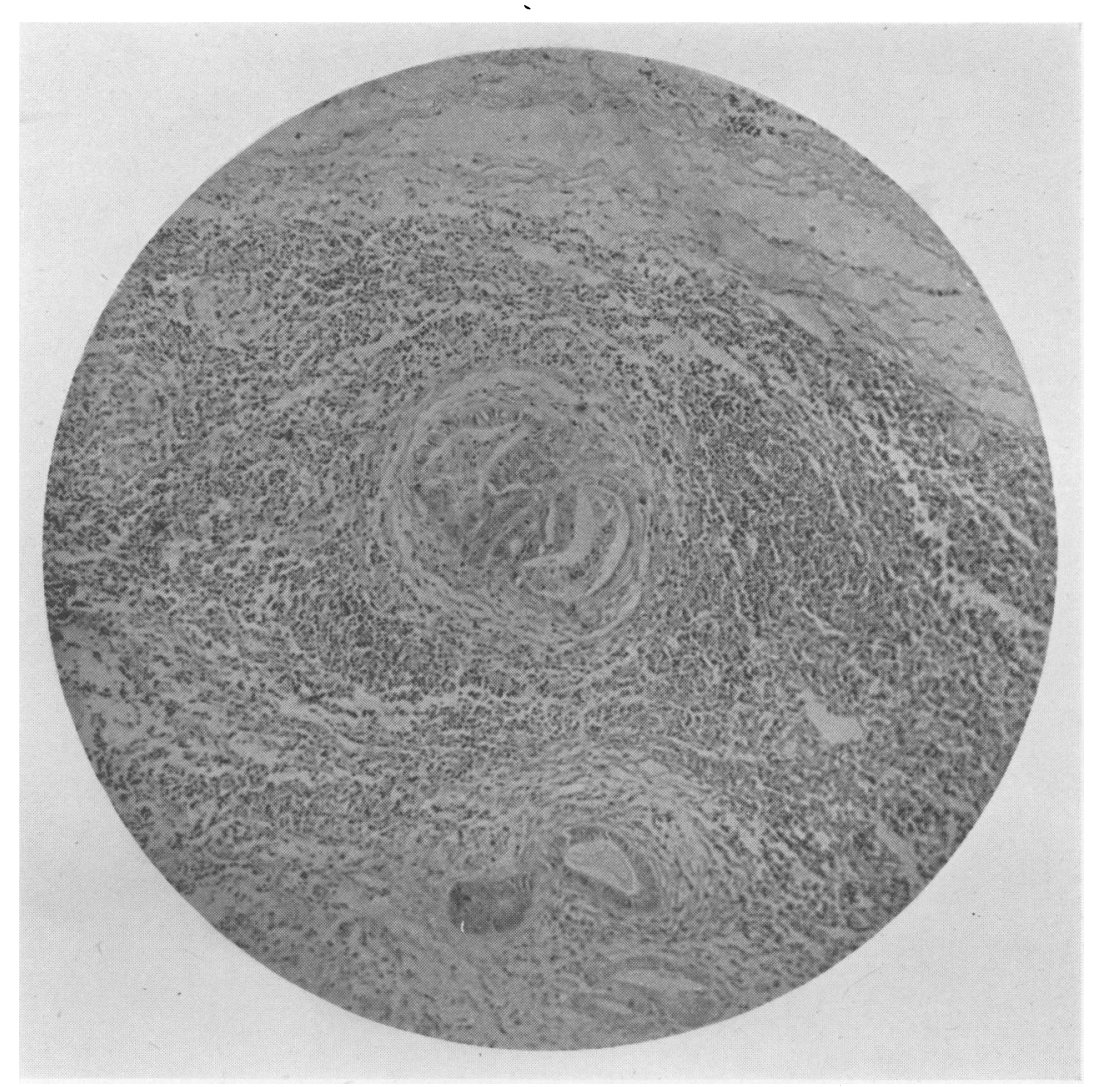

CARCINOMA OF RECTUM.-Microscopic section showing a small metastasis in a lymph node. 


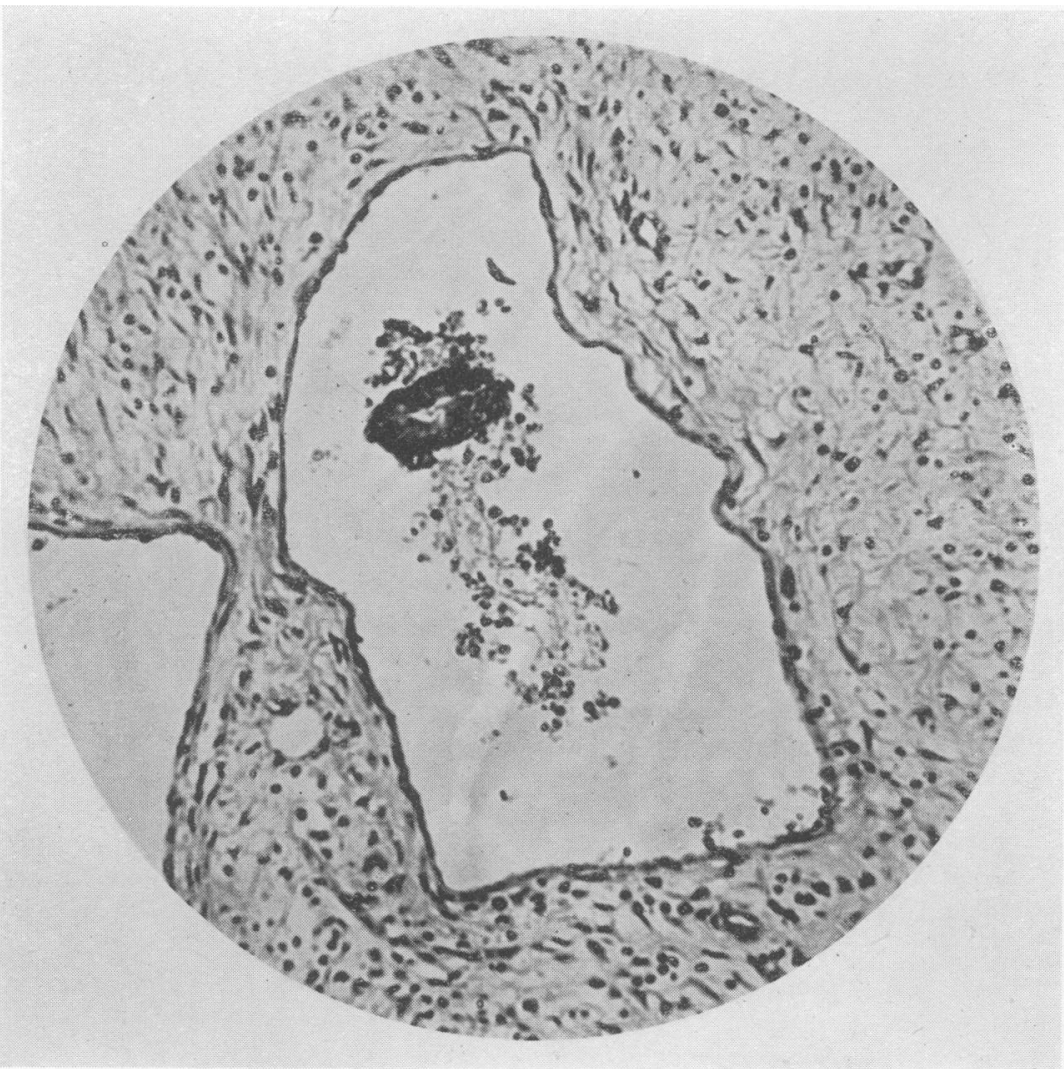

Carcinoma of ReCtum.-Microscopic section showing a malignant lymphatic embolus. 


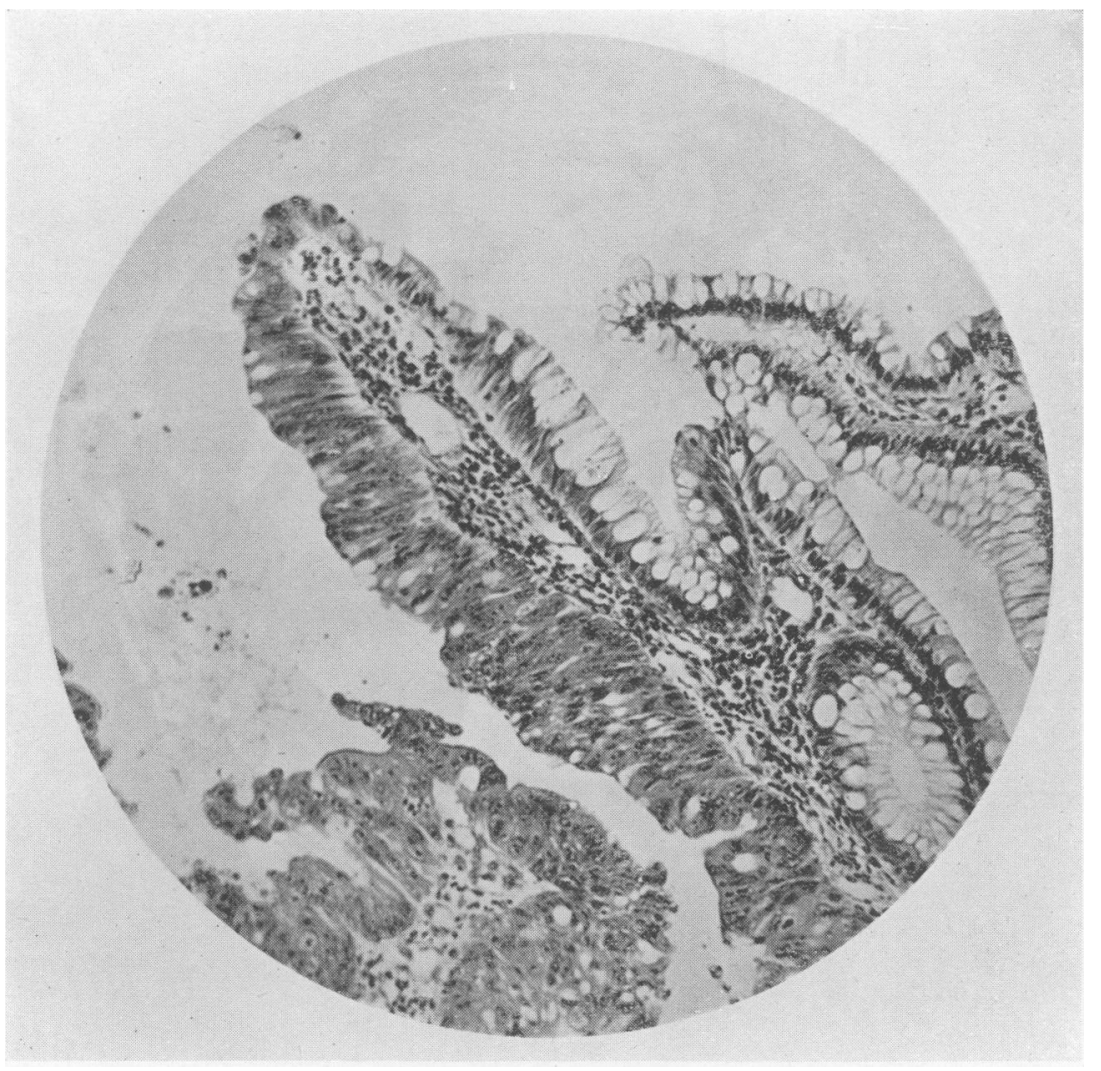

Carcinoma of ReCTum.-Microscopic section showing abrupt transition from normal to malignant rectal mucosa. 


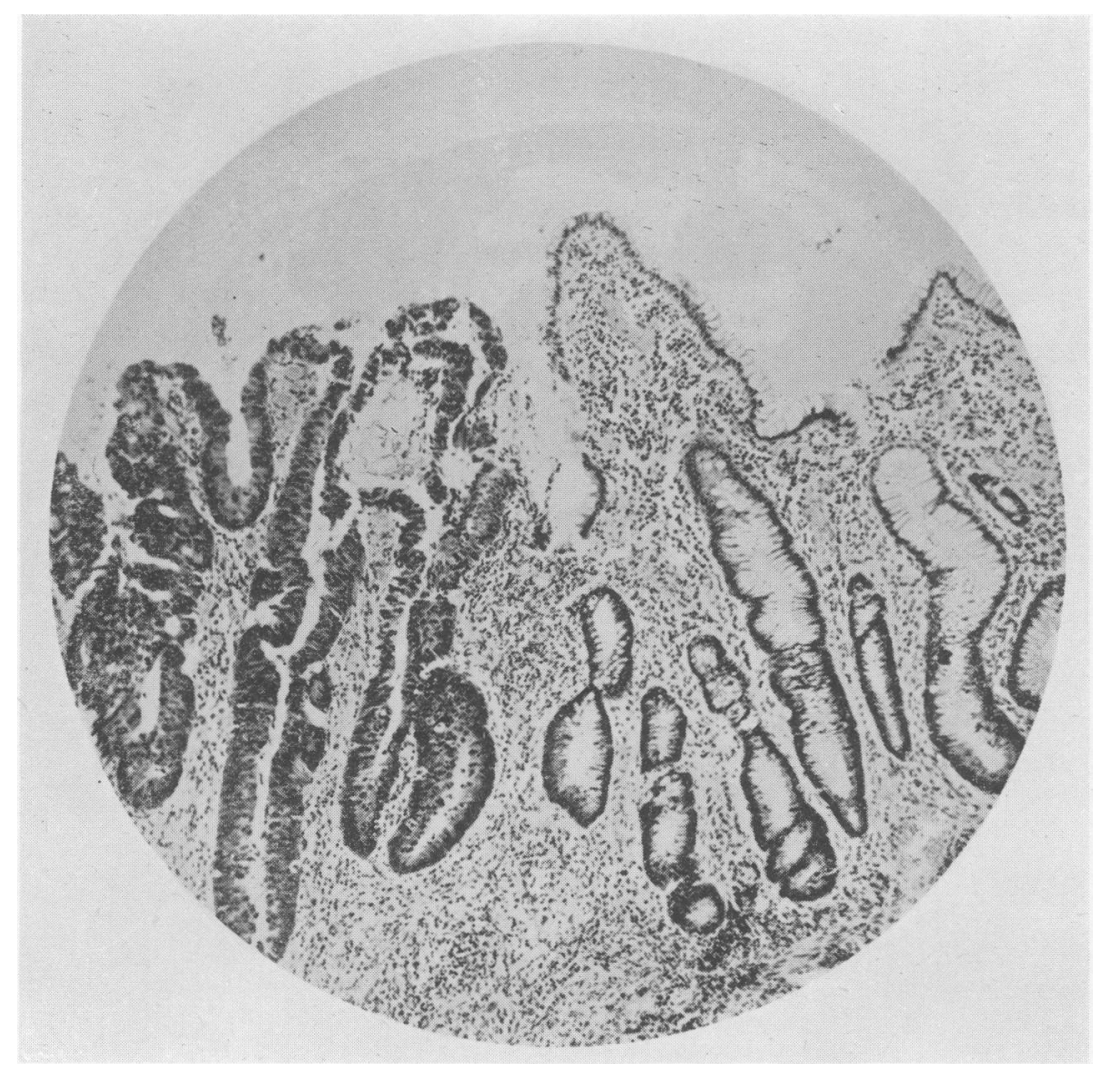

Carcinoma of Re(TUM.-Microscopic section showing abrupt transition from normal to malignant rectal mucosa. 


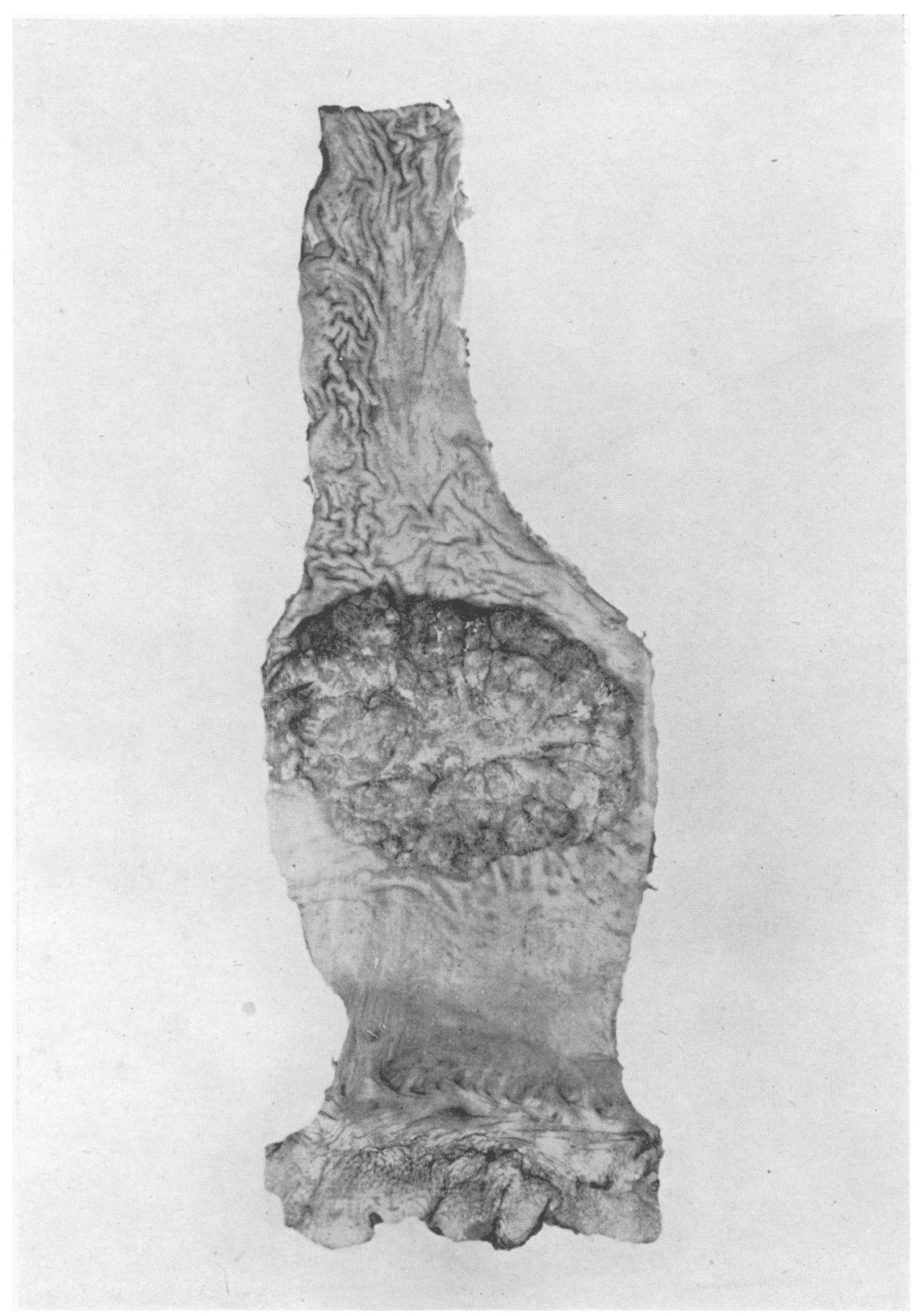

Carcinoma of the ReCtum.-Flat nodular type. (Museum of the Royal Cancer Hospital.) 


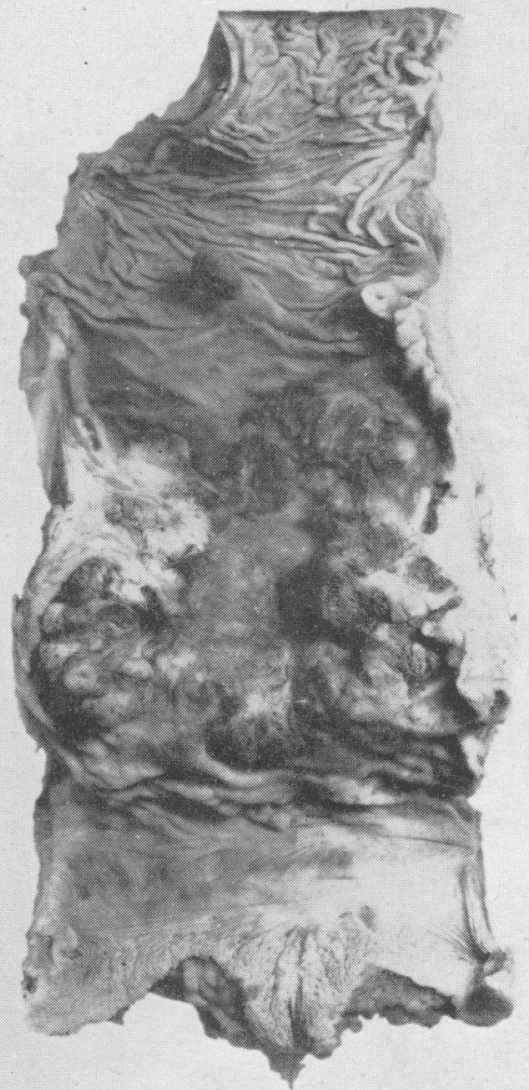

Carcinoma of THE RECTUM.-Annular fibro-carcinoma type.

(Museum of The Royal C ancer Hospital.) 


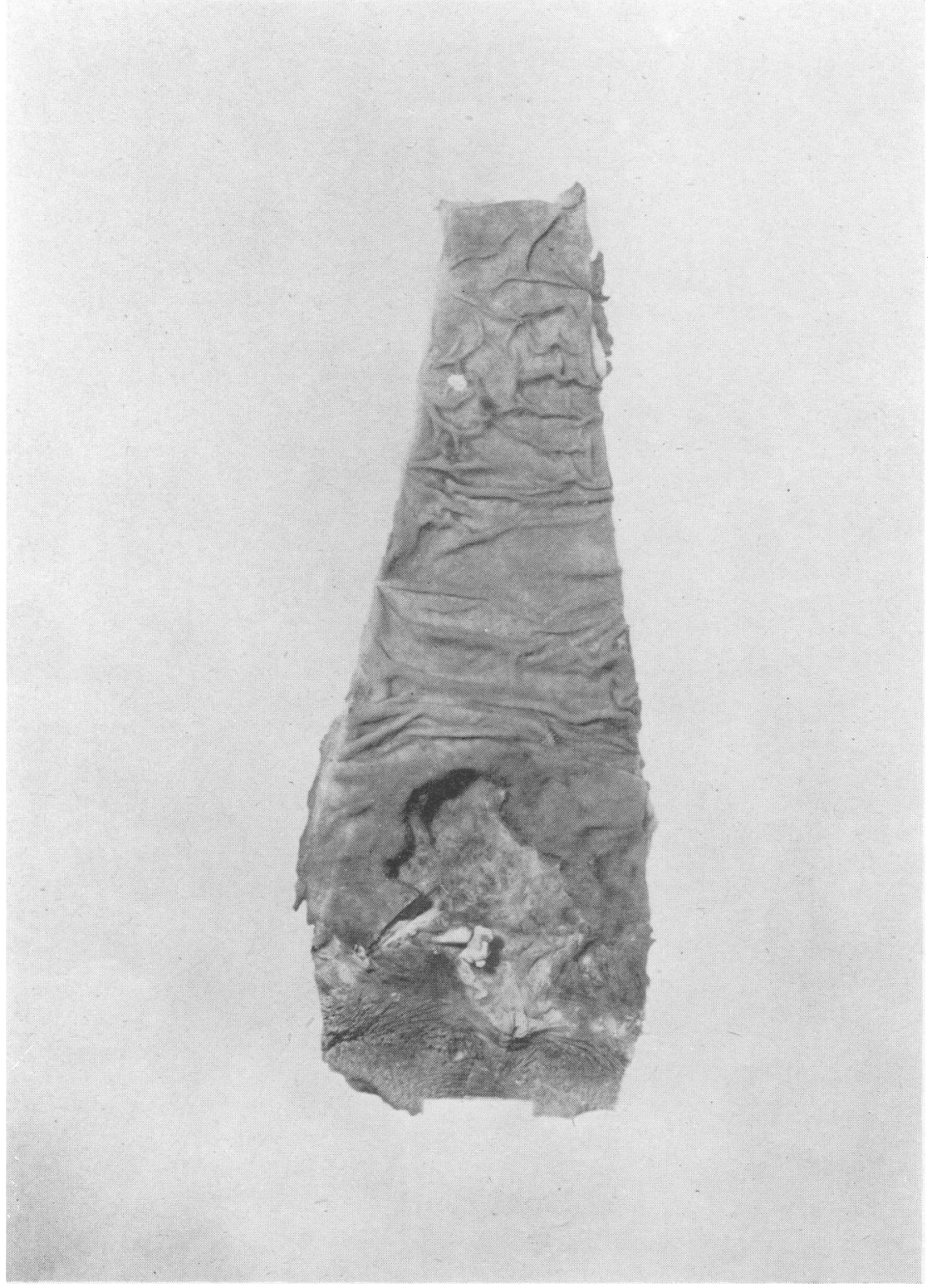




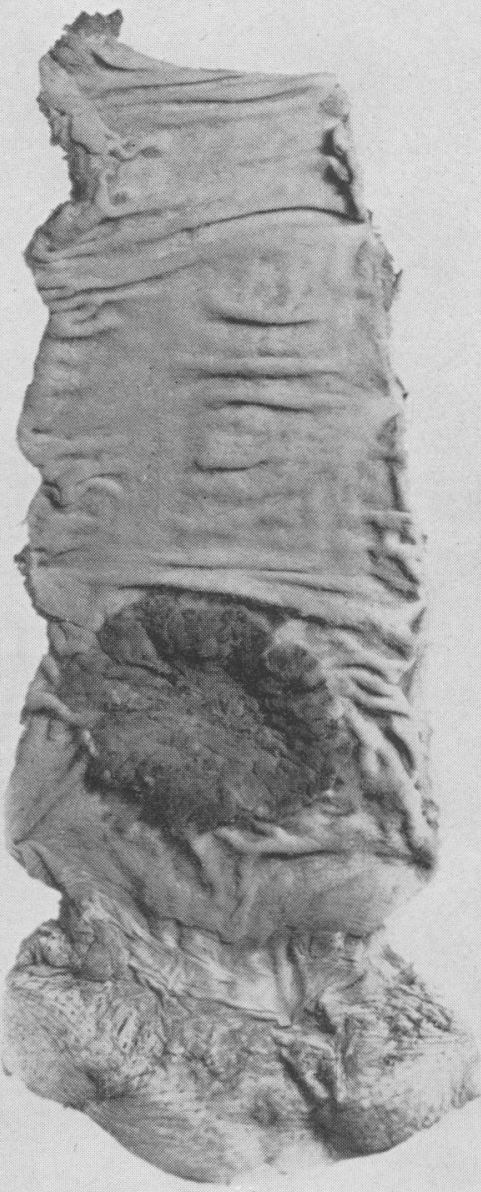




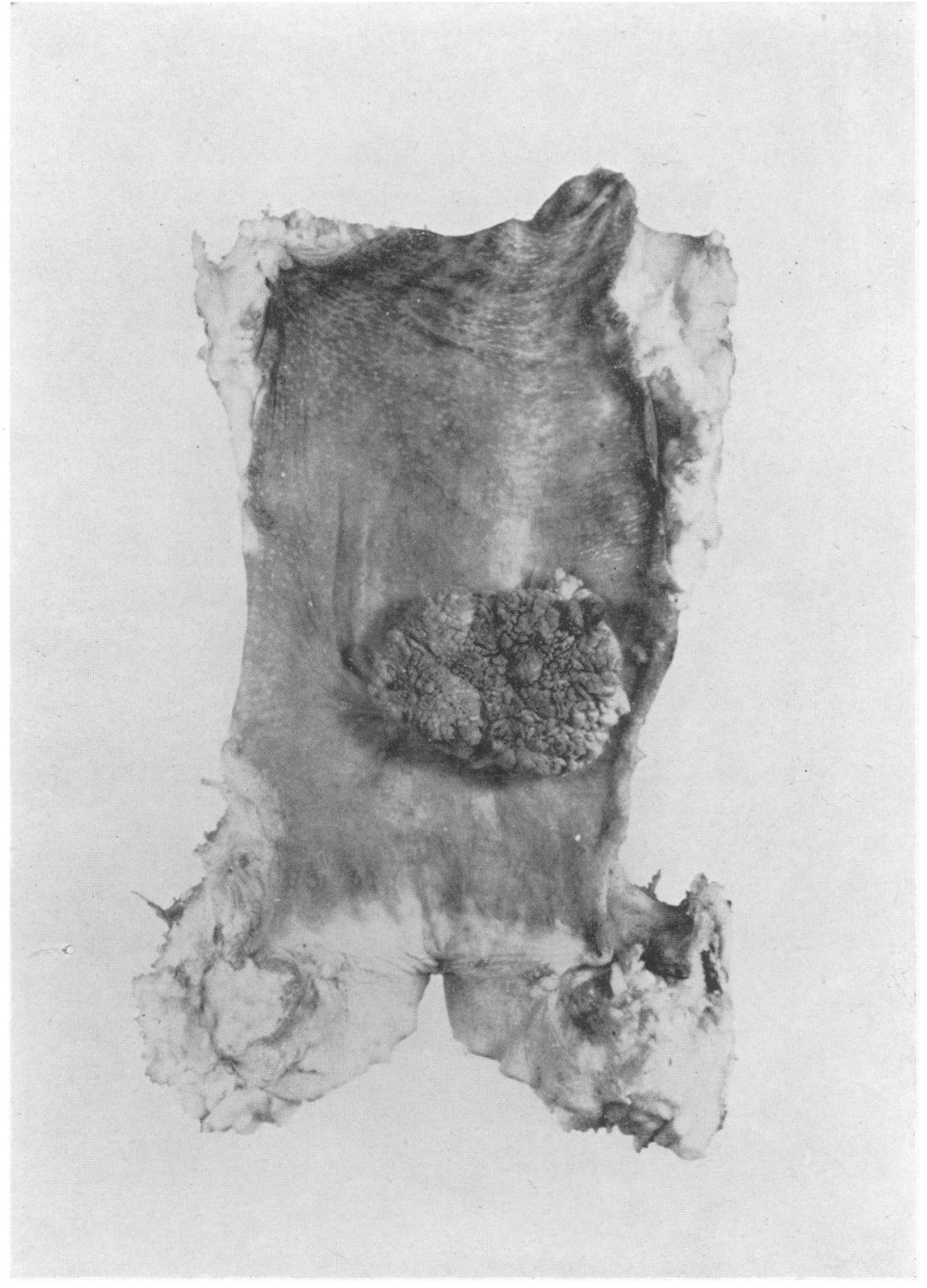

Papilloma of THE ReCtUM.

(Museum of the Royal Cancer Hospital.) 


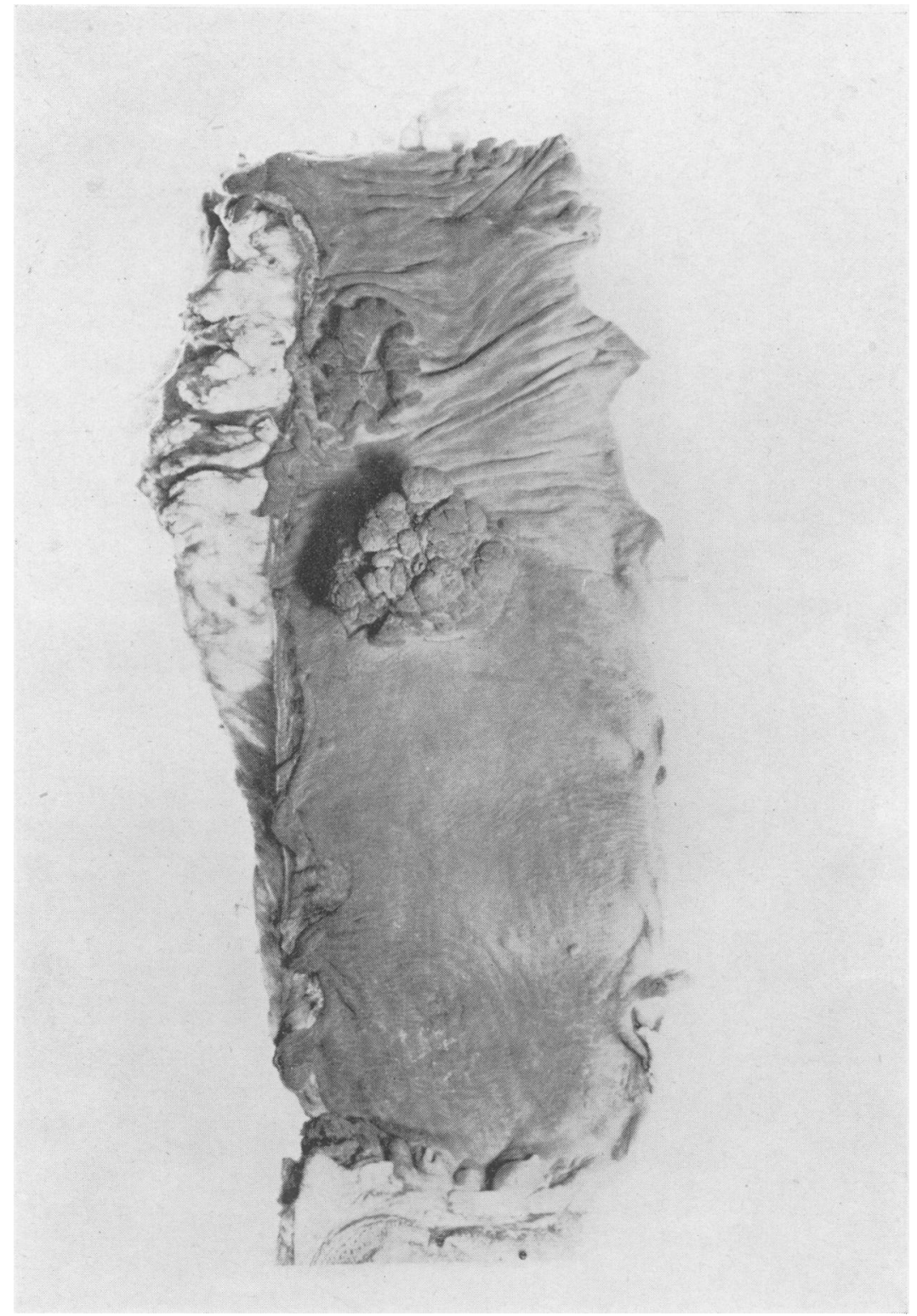




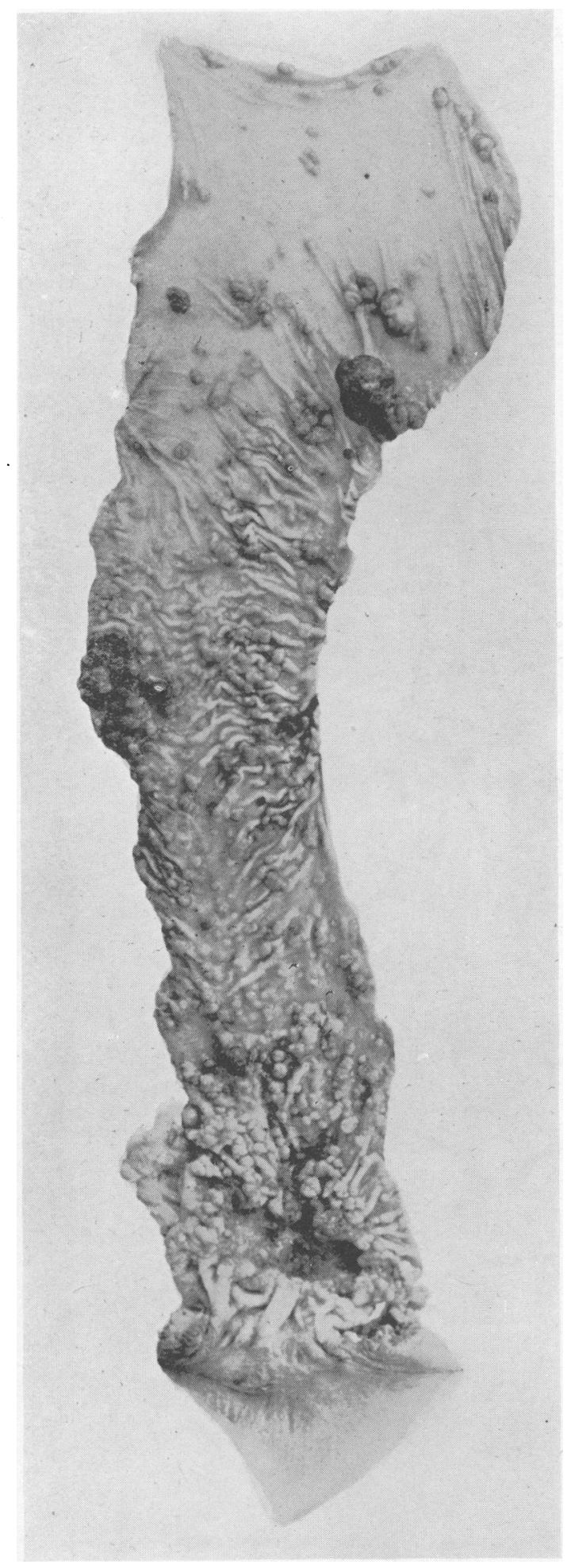

Polyposis coli with a carcinoma at the lower end of the rectum. 
Haemor rhoidal vessels bearing area

Ballooning of rectal ampulia

Affected

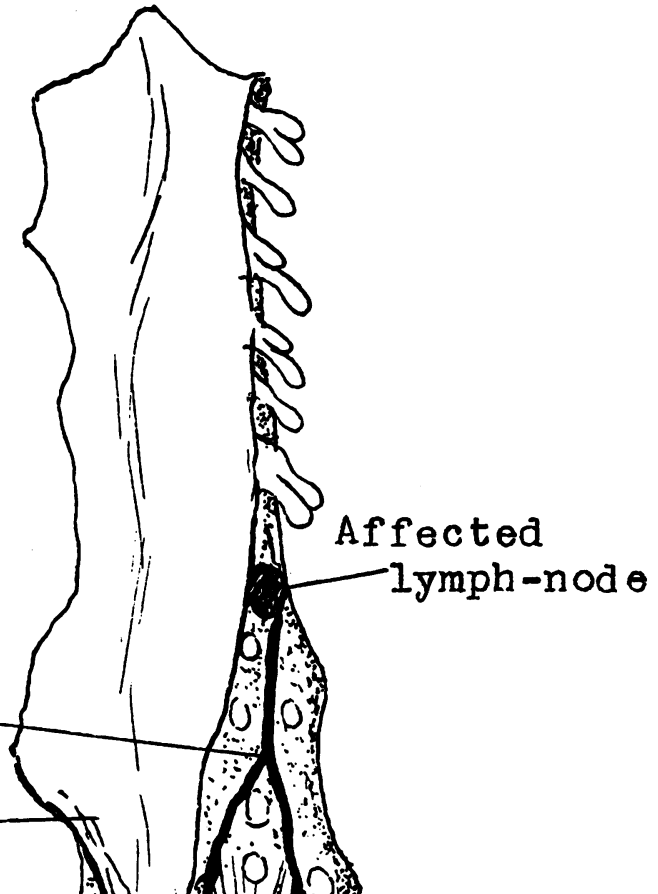

Carcinoma of RECTUM.-Schematic representation of specimen for pathological report.

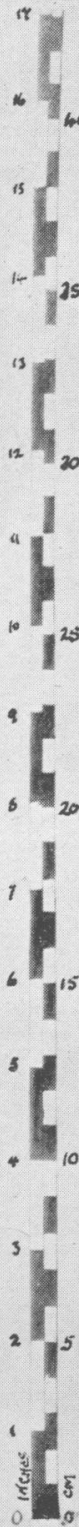


dehydration are present, they must be corrected together with any degree of secondary anaemia for which blood transfusion may be required. The cardiovascular system is examined, and the cardiac index is estimated; if this is less than 25 , the operation carries a considerable risk. The function of the kidneys is assessed and any oral sepsis is dealt with. Breathing exercises are instituted under the supervision of a physiotherapist. The patient's blood group is determined and cross-matching carried out with one or more donors. The diet should be of low-residue character and of high caloric value containing an adequate amount of protein, glucose and vitamins. A minimum amount of $100 \mathrm{gm}$. of protein should be taken daily. If the patient is apprehensive regarding the operation a sedative such as luminal (gr. $1 \frac{1}{2}$ ) should be prescribed. A laxative like senna is given and on alternate days a simple enema is administered until the day preceding the operation.

The use of sulfasuxidine. This substance was introduced in 194I by Poth and Knotts as an intestinal antiseptic and it is now widely used to modify the bacterial flora of the colon and to prepare the colon and rectum for operation. The gram-negative flora of the bowel are inhibited and both aerobic and anaerobic organisms are affected. The drug is given in doses of $3 \mathrm{gm}$. orally every four hours for seven days before the operation, if no observations are made on the coliform content of the faeces. Poth carried out quantitative bacteriological studies on the faeces of 350 patients which showed less than 1,000 coliform organisms per gram of wet faeces after the following periods of treatment :37 per cent. within three days of treatment; 80 per cent. within five days and 94 per cent. within seven days. If the coliform count is followed by the streak technique, Poth advises that the drug is given until the number of coliform colonies on the streaked plate is less than 20. The streaked technique is carried out as follows. A small particle of faeces is mixed with a drop of sterile water containing $5 \mathrm{mg}$. per cent. of para-aminobenzoic acid on a sterile slide and streaking a $2 \mathrm{~mm}$. loopful of this triturate on a desoxycholate or eosinmethylene blue agar plate. This procedure transfers approximately $0.01 \mathrm{gm}$. of faeces on to the plate. The plate count multiplied by Io0 gives the approximate number of coliform organisms per gram of faeces. If watery diarrhoea is present sulfathalidine $3.0 \mathrm{gm}$. every four hours is substituted for the sulfasuxidine; if the diarrhoea ceases the dose is reduced to $\mathrm{I} .5 \mathrm{gm}$.

These drugs are important adjuvants in the surgery of the colon and rectum and it is stressed that meticulous attention must still be given to surgical technique.

On the evening before the operation the last bowel wash-out is given and all the fluid must be syphoned away. The abdominal and perineal fields of operation are prepared in the usual way and sterile dressings are applied. One hour before the operation commences a hypodermic injection of omnopon (gr. I/3) and scopolamine (gr. I/150) is given. Shortly afterwards a gum-elastic coude catheter (No. 8) is passed so that the urinary bladder can be emptied completely when the patient is placed on the operation table. The catheter is closed with a sterile spigot and enclosed within a sterile towel. The patient is anaesthetized in bed with pentothal-sodium given intravenously, taken to the operating theatre and a spinal anaesthetic is then given unless this form of anaesthesia is contra-indicated. If desirable nitrous oxide and oxygen can be administered during the operation to keep the patient asleep. Before the operation is commenced the urinary bladder is emptied by applying suprapubic pressure while the catheter is removed slowly. In patients who are in poor general condition and spinal anaesthesia is contra-indicated, nitrous oxide, oxygen and ether anaesthesia should be used.

Post-operative care. When the operation is completed in the perineum a square of oiled silk is invaginated into the cavity which is packed lightly with rolled gauze. The laparotomy wound is covered with a double layer of gauze and sealed off with elastoplast in order to prevent contamination from the colostomy. The clamp is removed from the terminal colostomy which is thereby opened. The patient is transferred to a warmed bed brought to the operation theatre. During transport back to the ward all rough and unnecessary movements must be avoided together with any 
unnecessary exposure. If 'shock' is present the foot of the bed is elevated on blocks and resuscitation carried out. A blood transfusion of one pint is administered as a routine measure followed by glucose-saline intusion which is continued until peristalsis returns, normally after 48 to 72 hours. The blood is given at the rate of 30 drops each minute. For this purpose one ot the veins of the forearm is used so that the patient can be out of bed at an early stage. During the first 48 hours nothing is given by mouth and frequent rinsing is carried out. The patient is allowed to chew raw pineapple and chewing gum but fluid is not taken until active peristalsis returns when small quantities are given. For the relief of pain during the initial post-operative phase hypodermic injections of heroin (gr. I/I2) are given at intervals of four hours. The patient is instructed to carry out breathing exercises at frequent intervals and pulmonary ventilation may be increased by giving inhalations of a mixture of oxygen and carbon dioxide (5 per cent.) for periods of ten minutes at intervals of two hours. The position of the patient in bed must be changed so that he does not lie on the back for a period longer than six hours and frequent changes on to the side are made. The lower extremities are moved actively so that the calves of the legs do not press against the bed for long periods. Care is taken in lifting the patient, which is performed by placing arms unde $r$ the knees and round the posterior aspect of the chest.

At the end of eight hours following operation attention is given to the urinary bladder and the patient is encouraged to void urire. When mucturition cannot be performed normally, a catheter is passed under strict aseptic conditions at intervals of eight hours for a few days, the bladder being irrigated with a solution of mercury oxycyanide (strength I in 10,000) on each occasion.

After 24 hours have elapsed after operation, one-half of the gauze packing is removed from the perineal wound. In order that the reconstructed pelvic floor shall become a lining of the pelvis it is advisable for the patient to stand out of bed at this time, or as soon afterwards as the general condition will allow. At the end of 48 hours all the gauze packing and oiled-silk is removed. The perineal wound is then irrigated with a pint of hydrogen peroxide followed by a pint of a solution of mercury perchloride (strength $I$ in 500) and finally with a pint of a solution of normal saline. These irrigations are carried out using a douche-can and catheter. Care is taken to prevent the retention of exudate in the wound. At the end of the first week the solution of mercury perchloride is replaced by a solution of tincture of iodine (strength 2 drachms in I pint of water). From the third week onwards the solution of hydrogen peroxide and saline are omitted and the tincture of iodine is reduced to one-half strength. The sutures are removed from all the wounds on the tenth post-operative day; when Michel's clips are used for the laparotomy wound they are removed on the third post-operative day.

The colostomy may act spontaneously during the first few days, but in any case an aperient of either senna or cascara is given on the fourth post-operative day and on the fifth day and onwards the colostomy is washed out daily. During the initial 14 days this is performed with a pint of a solution of soft soap and substituted later by normal saline solution. The patient is instructed in colostomy management and after the morning wash-out no further attention is required during the ensuing day. On the tenth post-operative day, the colostomy is trimmed and any excessive length of bowel is removed so that the opening is on a level with the skin; no anaesthetic is required for this procedure. The index finger is passed into the colostomy every day to prevent cicatricial contraction occurring and measurements are taken for a colostomy belt which is then ordered.

\section{The Palliative Treatment}

It is an unfortunate fact that many patients still apply for treatment when the disease is so far advanced that only palliative treatment is possible. Owing to the large number in this group of patients this part of our subject is an important one and much can be done to ameliorate their symptoms which are often of a distressing nature.

The general management of the patient. Care is taken to preserve as far as possible the general health and the patient is placed under good hygienic conditions and given plenty of 
fresh air ; mild exercises are of value.

Diet. This must be of a nutritious nature and of high caloric value. A chart is maintained showing the amount of food taken and its caloric content; records are kept of the patient's body weight and of the serum protein content, these investigations being carried out at weekly intervals. The food is easily digested and of small residue with a caloric value of the order of 3,500 in 24 hours. The diet should contain creamed soups, creamed vegetables, chicken, mutton, beef, sweetbreads, liver, cereals, cheese and an adequate amount of sugar.

Care of the bowels. In the absence of obstructive symptoms a good bowel action should be achieved daily, the patient being given plenty of fluid and a daily dose of onehalf ounce of liquid paraffin. If obstructive symptoms are present salines are of value, and when faecal stasis occurs above a neoplasm enemata are required for relief. When the faecal mass is hard and dry, disintegration may be effected by an enema of hydrogen peroxide (strength 25 per cent.). If there is a tendency to form hard lumpy stools, or when diarrhoea and an excessive discharge of mucous are present, irrigation of the bowel is of value using a lotion composed of potassium permanganate (strength $I$ in 10,000 ) and lysol (strength $I$ in 200).

The relief of pain. The amount of pain experienced by these patients is variable and may be extremely severe. When it is relatively mild the weaker analgesics are used, such as aspirin, pyramidon and codeine. Later as the pain worsens morphine in adequate doses should be given. If the pain is situated in the sacro-coccygeal region, prostate, urinary bladder or vagina, suppositories containing morphine (gr. $\frac{1}{4}$ ) and belladonna (gr. $\frac{1}{2}$ ) are used. There are certain patients, however, who will not obtain sufficient relief from these measures and more requires to be done. Two additional methods of treatment for the relief of severe pain not controlled by analgesics are described.

The intrathecal injection of alcohol. The author has used this method of treatment in a number of patients with some measure of success so that it is well worthy of a trial. The technique is as follows :-the patient is placed in the left lateral position and the fourth lumbar interspace is defined. An injection of absolute alcohol is given into the subarachnoid space, the solution being injected drop by drop up to ten minims. The patient remains in the lett lateral position for a period of 20 minutes, and after this he is placed on his back for two hours. After the injection there may be mild motor effects and diminution in the tendon reflexes or these may be lost; zones of anaesthesia or hyperaesthesia may appear but all these effects are usually of a transitory nature and disappear after a few days at the most. There is no disturbance of bladder or bowel runctions in the average case although retention of urine has been reported. If no relief from pain follows this injection, it is repeated after the lapse of two weeks and on this occasion the patient is placed in the right lateral position. When relief from pain is achieved the benefit often lasts for a period of six months.

Cordotomy. In a small number of patients the pain is so severe and unrelieved by the methods of treatment described already that this operation is indicated. It is pointed out that in the case of a mid-line structure such as the rectum, it is necessary to section both spino-thalamic tracts leaving one segment of the spinal cord between these levels. The author has in mind one patient with an inoperable carcinoma of the rectum causing very severe pain unrelieved by all other methods, for whom he performed this operation, the spino-thalamic tracts being sectioned at the levels of D.4 and D.6 with complete relief from pain. After this operation every attention must be given to the prevention of pressure sores.

Insomnia. This may be a distressing symptom, not necessarily caused by the presence of pain. When this is the case various remedies are tried including allonal, medinal, luminal or bromides.

Secondary anaemia. There are several factors which contribute to the progressive secondary anaemia which is present in these patients. Iron and vitamin therapy is instituted and in certain patients a blood transfusion is helpful.

Apart from the general management of the patient thus outlined there are three operations 
of definite palliative value which are now described.

Palliative Radical Excision of the Rectum

There is a group of patients with carcinoma of the rectum giving rise to hepatic metastases, but the local lesion can be removed. The argument is advanced that by removing the rectum the patient is made more comfortable in that a bleeding, discharging, ulcerating neoplasm is no longer present and the possibility of further local complications developing is obviated. Thus it is justifiable to perform a palliative radical excision of the rectum in these cases providing it is judged that the patient is fit enough for the operation and possesses a reasonable period of expectation of life. The patients must be chosen carefully and in some the final decision will be made at exploratory laparotomy. By this means a great deal of suffering will be ameliorated.

\section{Colostomy}

This is a valuable method of treatment in advanced carcinoma of the rectum for several reasons. Relief is afforded those patients who have developed various degrees of intestinal obstruction or when fistulae-recto-vesical, recto-vaginal or vesico-colic-have occurred. If colostomy is performed many distressing local complications will be averted, especially of an inflammatory nature. Further, it is found there is often a temporary improvement in the patient's general health, for more sleep is obtained, an increased diet is taken and a better evacuation of the bowel is achieved. The question must be answered concerning the time at which colostomy should be performed, and it is laid down that when a carcinoma of the rectum is considered to be inoperable, the sooner colostomy is carried out the better. The words of Miles should be remembered when he said, 'from what I have seen of these cases I am convinced that, as soon as carcinoma of the rectum is found to be inoperable, every day lost before resorting to colostomy is a day to the bad.' With modern management, a colostomy is no longer an intolerable burden for the patient to carry, and he is given full instructions concerning the subject before leaving hospital.

Diathermy of the carcinoma. A very distressing symptom seen frequently in patients with advanced carcinoma of the rectum is excessive discharge of blood and mucous. It is found that destruction of the projecting, fungating portion of the neoplasm is a valuable method of palliation in these cases. The operation is performed through a sigmoidoscope and the object of the procedure is to transform a large fungating growth into a flat ulcer. If the symptoms recur at a later date the operation can be repeated.

Irradiation treatment. Over a period of a number of years investigations cor.cerning the value of irradiation treatment in carcinoma of the rectum have been carried out. Several methods of treatment have been tried including irradiation by means of radon seeds, radium needles and high voltage $\mathrm{X}$-irradiation. The results achieved in columnar celled adenocarcinoma of the rectum have been disappointing.

I wish to record my thanks to Dr. Woodhouse Price, Pathologist to the Royal Cancer Hospital for help with the illustrations.

REFERENCES

I. DUKES, CUTHBERT F. (1940), Cancer of the Rectum, An Analysis of 1,000 cases, four. Path. and Bact., 50, No. 3, 527.

RAVEN, RONALD W. (I939), Early Carcinoma of the Rectum, Proc. Roy. Soc. Med., 32, 907.

3. GABRIEL, W. B., The Principles and Practice of Rectal Surgery, London, p. 263.

4. NORBURY, L. E. C. (1930-3I), Multiple Primary Malignant Growths, with Special Reference to the Colon and Rectum,

Proc. Roy. Soc. Med., 24, Pt. I, 198.

5. MILES, W. ERNEST (1939), Rectal Surgery, London, p. 244.

\section{RUTHIN CASTLE, NORTH WALES}

A Clinic for the diagnosis and treatment of Internal Diseases (except Mental or Infectious Diseases). The Clinic is provided with a staff. of doctors, technicians and nurses.

The surroundings are beautiful. The climate is mild. There is central heating throughout. The annual rainfall is $\mathbf{3 0 . 5}$ inches, that is, less than the average for England.

The Fees are inclusive and vary according to the room occupied.

For particulars apply to THE SECRETARY, Ruthin Castle, North Wales.

Telegrams : Castle, Ruthin.

Telephone : Ruthin 66. 